\title{
Halogenation of 1-alkyl-7-azaisatins using $N$-halosuccinimides: regioselective synthesis of 1-alkyl-5-halo-7-azaisatins
}

\author{
Jiro Tatsugi,* Tong Zhiwei, Yukio Tsuchiya, and Takayoshi Ito \\ Department of Applied Chemistry, Faculty of Engineering, Aichi Institute of Technology, \\ Yachigusa, Yakusa-Cho, Toyota 470-0392, Japan \\ E-mail:jtatsugi@aitech.ac.jp
}

\begin{abstract}
A facile and regioselective protocol for the halogenation of 1-alkyl-7-azaisatins with NBS or NCS in aprotic solvents under mild conditions to synthesize 1-alkyl-5-halo-7-azaisatins was developed.
\end{abstract}

Keywords: 1-Alkyl-7-azaisatins, 1-alkyl-5-halo-7-azaisatins, NBS, NCS

\section{Introduction}

In recent years azaindoles have received considerable interest in view of their potential relationship to pharmacologically important indoles and purine nucleus, because of their interesting biological activities. There are only a few azaindole derivatives in nature, so many azaindoles are synthetically prepared for the development of pharmaceutical agents. Their syntheses and properties have been surveyed comprehensively in reviews. ${ }^{1}$ On the other hand, for 7-azaisatins only a few compounds are known, 7-azaisatin, 5-bromo-7-azaisatin and 1-alkyl7-azaisatins. 7-Azaisatin was first obtained by treatment of 7-azaoxindole with nitrous acid to give its 3-oxime, followed by hydrolysis of the oxime. ${ }^{2}$ Another route for the preparation of 7 azaisatin from 7-azaindole in five steps with difficulty was reported by Parrick and coworker ${ }^{3}$ in 1989. 5-Bromo-7-azaisatin, an intermediate to synthesize 5-bromo-7-azaoxindole derivative which was the first non peptide antagonist of Bombesin, was synthesized by oxidation of 7azaindole with $\mathrm{CrO}_{3}$ as oxidant in the presence of bromine. ${ }^{4}$ Recently we have reported one-pot synthesis of 1-alkyl-7-azaisatins via 1-alkyl-7-azaindoles. ${ }^{5}$ In the course of our studies on the photochemical behavior of cyclic vicinal polycarbonyl compounds, ${ }^{6}$ we need to prepare halogenated 1-alkyl-7-azaisatins as structurally related compounds to investigate the photoreactions of heterocyclic vicinal polycarbonyl compounds. In this paper we wish to 
describe a facile and regioselective method for synthesis of 1-alkyl-5-halo-7-azaisatins with NBS or NCS in aprotic solvents, DMSO and DMF.

\section{Results and Discussion}

Recently we have developed new and facile methods for the preparation of acenaphthenequinones, ${ }^{7}$ benzils, ${ }^{8}$ 1,2,3-indanetriones, ${ }^{9}$ and 1 -alkyl-7-azaisatins. ${ }^{5}$ When the mixture of 1 methyl-7-azaindole with 2.5 equivalents of NBS in DMSO was allowed to react at $80^{\circ} \mathrm{C}$ for over $100 \mathrm{~h}$ under reduced pressure, 1-methyl-7-azaisatin (1a) was obtained in good yield along with a brominated product (2a) as a result of bromination of $\mathbf{1 a}$ in low yield. These results suggest that bromination of 1a may also take place under our conditions. So, in order to obtain 2a we examined the reaction of 1-methyl-7-azaindole with 3.0 equivalents of NBS in DMSO under similar conditions and found that $\mathbf{2 a}$ was obtained in a moderate yield. When the bromination of $1 \mathrm{a}$ with 1.3-equivalents of NBS in DMF under mild conditions at 50 for $41 \mathrm{~h}$ was carried out, the brominated product $2 \mathrm{a}$ was obtained in good yield. It shows two doublet peaks at $\delta 7.95(1 \mathrm{H})$ and $8.55(1 \mathrm{H})$ in the ${ }^{1} \mathrm{H}$ NMR spectrum and 240 and 242 molecular ion peak with similar intensity in the MS spectrum. As outlined in Scheme 1 and Table 1, the reaction of 1-alkyl-7azaisatins (1a-c) with NBS in DMF gave 1-alkyl-5-bromo-7-azaisatins (2a-c) in good yields. ${ }^{10}$<smiles>[R]N1C(=O)C(=O)c2cccnc21</smiles>

$1 a, b, c$

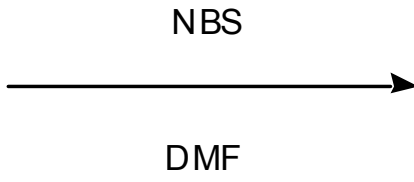

a: $R=M e, b: R=E t, c: R=B n$

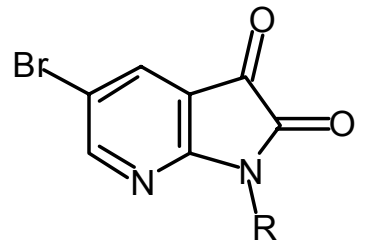

$2 a, b, c$

\section{Scheme 1}

Some representative results are summarized in Table 1, and the following procedure is typical: a mixture of $\mathbf{1}$ and NBS or NCS in DMF was heated to 50 for about two days. After cooling to room temperature the reaction mixture was poured into cold water, extracted with dichloromethane, the extract dried over magnesium sulfate, and the solvent evaporated under reduced pressure to give the crude product. 1-Methyl-5-bromo-7-azaisatin 2a was obtained after purification with chromatography and then recrystallization from dichloromethane. Other 1alkyl-7-azaisatins $\mathbf{1 b}, \mathbf{c}$ were reacted analogously to give 5-bromo derivatives $\mathbf{2 b , c}$ in $83-86 \%$ yields. As shown in Scheme 2 the similar reaction of 1a-c with NCS in DMF gave 1-alkyl-5chloro-7-azaisatin (3a-c) in 66-79 yields. 
<smiles>[R]N1C(=O)C(=O)c2cccnc21</smiles>

1a,b,c

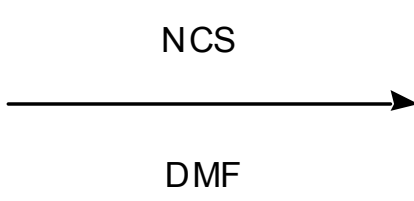

a: $R=M e, b: R=E t, c: R=B n$<smiles>[R]N1C(=O)C(=O)c2cc(Cl)cnc21</smiles>

$3 a, b, c$

\section{Scheme 2}

Table 1. Halogenation of 1-alkyl-7-azaisatins with NBS or NCS in DMF

\begin{tabular}{|c|c|c|c|}
\hline \multirow[t]{2}{*}{ Compounds } & \multirow[t]{2}{*}{$N$-Halosuccinimide } & \multicolumn{2}{|c|}{ Yields $(\%)$} \\
\hline & & $2 a-c$ & $3 a-c$ \\
\hline $1 \mathbf{a}$ & NBS & 73 & \\
\hline $1 \mathbf{a}$ & NCS & & 78 \\
\hline $1 b$ & NBS & 83 & \\
\hline $1 b$ & NCS & & 66 \\
\hline 1c & NBS & 86 & \\
\hline $1 \mathrm{c}$ & NCS & & 79 \\
\hline
\end{tabular}

The structures of novel products were assigned based on the IR, ${ }^{1} \mathrm{H}$ NMR and ${ }^{13} \mathrm{C} N M R$, and MS spectra. The ${ }^{1} \mathrm{H}$ NMR spectra of compounds 2 and $\mathbf{3}$ showed two doublet peaks at $\delta$ 7.78$7.92 \mathrm{ppm}$ for the proton of 6 position and $\delta 8.42-8.52 \mathrm{ppm}$ for the proton of 4 position of the pyridine respectively. From the results, we assigned the structure of $\mathbf{2}$ and $\mathbf{3}$ as 1-alkyl-5-halo-7azaisatins. The ${ }^{13} \mathrm{C}$ NMR spectra were in agreement with the proposed structures (see Experimental Section).

In conclusion, we have developed the versatile, readily method for the synthesis of 1-alkyl-5halo-7-azaisatins from the corresponding 1-alkyl-7-azaisatins with NBS or NCS under mild conditions.

\section{Experimental Section}

General Procedures. Melting points were determined on a Yanagimoto hot-stage apparatus and are uncorrected. IR spectra were recorded on a Nihon Bunko 7300 FT-IR or a Shimadzu FTIR4800S spectrometer in $\mathrm{KBr}$ with absorptions in $\mathrm{cm}^{-1}$. The UV-VIS spectra were recorded using a Shimadzu UV-3100S or a Shimadzu UV-2450 spectrophotometer. ${ }^{1} \mathrm{H}$ and ${ }^{13} \mathrm{C}$ NMR spectra were recorded on Varian Genimi-200 from a solution $\mathrm{CDCl}_{3}$ of the product. ${ }^{1} \mathrm{H}$ chemical shifts are expressed as $\delta$ values (ppm) relative to TMS as an internal standard. MS spectra and HRMS were recorded on Hitachi 80-B spectrometer. For column chromatography, silica gel (nacalai 
tesque, 230 - 400 mesh) was used. Solvents were dried and purified in the usual manner. 7Azaindole (Aldrich), NBS and NCS were commercially available and were used without purification. 1-Methyl-, 1-ethyl- and 1-benzyl-7-azaisatins were prepared from 7-azaindole according to the previously reported procedure. ${ }^{5}$

\section{Bromination of 1-alkyl-7-azaisatins. General procedure}

To a solution of 1-methyl-7-azaisatin (1a) $(0.10 \mathrm{~g}, 0.6 \mathrm{mmol})$ in DMF $(5 \mathrm{~mL})$ was added NBS $(0.16 \mathrm{~g}, 0.9 \mathrm{mmol})$ at $\mathrm{r}$. $\mathrm{t}$. The solution was stirred at $\mathrm{r}$. $\mathrm{t}$ for $4 \mathrm{~h}$ and then at $50{ }^{\circ} \mathrm{C}$ for $41 \mathrm{~h}$. The progress of the reaction was monitored by GC and GC-MS. After disappearance of 1a, the reaction mixture was poured into water $(30 \mathrm{~mL})$, followed by extracting with dichloromethane (10 mL x 3). The combined extracts were washed three times with distilled water and dried $\left(\mathrm{MgSO}_{4}\right)$. After removal of the solvent, the residue was purified chromatography on silica gel with dichloromethane as an eluent to give the pure product.

5-Bromo-1-methyl-7-azaisatin (2a). Yield = 73\%, yellow plates; mp $171-172{ }^{\circ} \mathrm{C}\left(\mathrm{CH}_{2} \mathrm{Cl}_{2}\right)$; IR $(\mathrm{KBr}) v=1752(\mathrm{C}=\mathrm{O}), 1603,1586,1466 \mathrm{~cm}^{-1}$; UV $(\mathrm{MeOH}) \lambda \max (\varepsilon)=266.5 \mathrm{~nm}(17930) ;{ }^{1} \mathrm{H} \mathrm{NMR}$ $\left(\mathrm{CDCl}_{3}\right) \delta: 3.55\left(\mathrm{~s}, 3 \mathrm{H}, \mathrm{CH}_{3}\right), 7.95(\mathrm{~d}, 1 \mathrm{H}, J=2.3 \mathrm{~Hz}, 4-\mathrm{H}), 8.55(\mathrm{~d}, 1 \mathrm{H}, J=2.3 \mathrm{~Hz}, 6-\mathrm{H}) ;{ }^{13} \mathrm{C} \mathrm{NMR} \delta$ : 25.2, 112.7, 115.1, 135.0, 156.3, 157.7, 162.1, 180.8; MS m/z $(\%)=240\left(\mathrm{M}^{+}, 91\right), 242\left(\mathrm{M}^{+}+2\right.$, 90), 212 (71), 214(70), 184 (74), 186 (73), 157 (100), 159 (98); HRMS Calcd for $\mathrm{C}_{8} \mathrm{H}_{5} \mathrm{~N}_{2} \mathrm{O}_{2} \mathrm{Br}$ 239.9535 found 239.9562 and 241.9515 found 241.9530 .

5-Bromo-1-ethyl-7-azaisatin (2b). Yield = 83\%, yellow plates; mp $142-143{ }^{\circ} \mathrm{C}\left(\mathrm{CH}_{2} \mathrm{Cl}_{2}\right)$; IR $(\mathrm{KBr}) v=1752(\mathrm{C}=\mathrm{O}) \mathrm{cm}^{-1}$; UV $(\mathrm{MeOH}) \lambda \max (\varepsilon)=267 \mathrm{~nm}(10820) ;{ }^{1} \mathrm{H} \mathrm{NMR}\left(\mathrm{CDCl}_{3}\right) \delta: 1.33$ (t, 3H, $J=7.2 \mathrm{~Hz}, \mathrm{CH}_{3}$ ), 3.91 (q, 2H, $J=7.2 \mathrm{~Hz}, \mathrm{CH}_{2}$ ), 7.92 (d, 1H, $\left.J=2.3 \mathrm{~Hz}, 4-\mathrm{H}\right), 8.52$ (d, $1 \mathrm{H}, J=2.3 \mathrm{~Hz}, 6-\mathrm{H}) ;{ }^{13} \mathrm{C}$ NMR $\delta: 12.8,34.4,112.8,115.0,135.1,156.3,157.4,162.9,181.2$; $\mathrm{MS} \mathrm{m} / \mathrm{z}(\%)=254\left(\mathrm{M}^{+}, 93\right), 256\left(\mathrm{M}^{+}+2,90\right), 225$ (5), 227(5), 197 (95), 199 (100), 171 (36), 173 (36), 155 (43), 157 (38); HRMS Calcd for $\mathrm{C}_{9} \mathrm{H}_{7} \mathrm{~N}_{2} \mathrm{O}_{2} \mathrm{Br} 253.9691$ found 253.9714 and 255.9671 found 255.9717.

1-Benzyl-5-bromo-7-azaisatin (2c). Yield = 86\%, yellow plates; mp $153-154{ }^{\circ} \mathrm{C}\left(\mathrm{CH}_{2} \mathrm{Cl}_{2}\right)$; IR $(\mathrm{KBr}) v=1751(\mathrm{C}=\mathrm{O}) \mathrm{cm}^{-1} ; \mathrm{UV}\left(\mathrm{CH}_{2} \mathrm{Cl}_{2}\right) \lambda \max (\varepsilon)=277.5 \mathrm{~nm}(4793) ;{ }^{1} \mathrm{H} \mathrm{NMR}\left(\mathrm{CDCl}_{3}\right) \delta$ :4.99 (s, 2H, $\left.\mathrm{CH}_{2}\right), 7.2$-.7.5 (m 5H), 7.89 (d, 1H, $\left.J=2.4 \mathrm{~Hz}, 4-\mathrm{H}\right), 8.52(\mathrm{~d}, 1 \mathrm{H}, J=2.4 \mathrm{~Hz}, 6-\mathrm{H})$; ${ }^{13} \mathrm{C}$ NMR $\delta: 42.8,112.8,115.2,128.2,128.8,128.9,129.0,135.1,154.1,157.4,161.7,180.8$; $\mathrm{MS} \mathrm{m} / \mathrm{z}(\%)=316\left(\mathrm{M}^{+}, 19\right), 318\left(\mathrm{M}^{+}+2,19\right), 288$ (5), $290(5), 259$ (33), 261 (33), 169 (10), 171 (10), 91 (100); HRMS Calcd for $\mathrm{C}_{14} \mathrm{H}_{9} \mathrm{~N}_{2} \mathrm{O}_{2} \mathrm{Br} 315.9846$ found 315.9816 and 317.9826 found 317.9812 .

5-Chloro-1-methyl-7-azaisatin (3a). Yield = 78\%, yellow plates; mp $157-158{ }^{\circ} \mathrm{C}\left(\mathrm{CH}_{2} \mathrm{Cl}_{2}\right)$; IR $(\mathrm{KBr}) v=1753(\mathrm{C}=\mathrm{O}) \mathrm{cm}^{-1}$; UV $(\mathrm{MeOH}) \lambda \max (\varepsilon)=265.5 \mathrm{~nm}(11880), 406.5(350) ;{ }^{1} \mathrm{H} \mathrm{NMR}$ $\left(\mathrm{CDCl}_{3}\right) \delta: 3.33\left(\mathrm{~s}, 3 \mathrm{H}, \mathrm{CH}_{3}\right), 7.79(\mathrm{~d}, 1 \mathrm{H}, J=2.4 \mathrm{~Hz}, 4-\mathrm{H}), 8.42(\mathrm{~d}, 1 \mathrm{H}, J=2.4 \mathrm{~Hz}, 6-\mathrm{H}) ;{ }^{13} \mathrm{C}$ NMR $\delta: 25.2,112.2,119.7,134.2,154.2,157.8,161.8,181.0 ; \mathrm{MS} \mathrm{m} / \mathrm{z}(\%)=196\left(\mathrm{M}^{+}, 86\right)$, 198( $\left.\mathrm{M}^{+}+2,20\right), 168$ (52), 170(10), 140 (76), 142 (18), 112 (100), 114 (30), 74 (24); HRMS Calcd for $\mathrm{C}_{8} \mathrm{H}_{5} \mathrm{~N}_{2} \mathrm{O}_{2} \mathrm{Cl} 196.0038$ found 196.0015 and 198.0010 found 198.0018 . 
5-Chloro-1-ethyl-7-azaisatin (3b). Yield $=66 \%$, yellow plates; mp $124-126{ }^{\circ} \mathrm{C}\left(\mathrm{CH}_{2} \mathrm{Cl}_{2}\right)$; IR $(\mathrm{KBr}) \mathrm{v}=1748(\mathrm{C}=\mathrm{O}) \mathrm{cm}^{-1}$; UV $\left(\mathrm{CH}_{2} \mathrm{Cl}_{2}\right) \lambda \max (\varepsilon)=287 \mathrm{~nm}$ (9032), 322.5 (3664) nm; ${ }^{1} \mathrm{H}$ NMR $\left(\mathrm{CDCl}_{3}\right) \delta: 1.34\left(\mathrm{t}, 3 \mathrm{H}, J=7.3 \mathrm{~Hz}, \mathrm{CH}_{3}\right), 3.92\left(\mathrm{q}, 2 \mathrm{H}, J=7.3 \mathrm{~Hz}, \mathrm{CH}_{2}\right), 7.81(\mathrm{~d}, 1 \mathrm{H}, J=2.4 \mathrm{~Hz}, 4-$ H), 8.43(d, $1 \mathrm{H}, J=2.4 \mathrm{~Hz}, 6-\mathrm{H}) ;{ }^{13} \mathrm{C}$ NMR $\delta: 12.7,34.3,112.2,127.5,132.4,154.0,157.5$, 161.5, 181.2; MS m/z (\%) = 210( $\left.\mathrm{M}^{+}, 71\right), 212\left(\mathrm{M}^{+}+2,19\right), 181(6), 183(1), 153$ (100), 155 (36), 112 (48), 114 (7), 73 (20); HRMS Calcd for $\mathrm{C}_{9} \mathrm{H}_{7} \mathrm{~N}_{2} \mathrm{O}_{2} \mathrm{Cl} 210.0195$ found 210.0185 and 212.0165 found 212.0118 .

1-Benzyl-5-chloro-7-azaisatin (3c). Yield $=79 \%$, yellow plates; mp $126-128{ }^{\circ} \mathrm{C}\left(\mathrm{CH}_{2} \mathrm{Cl}_{2}\right)$; IR $(\mathrm{KBr}) v=1753(\mathrm{C}=\mathrm{O}) \mathrm{cm}^{-1}$; UV $(\mathrm{MeOH}) \lambda \max (\varepsilon)=264.0 \mathrm{~nm}(10260), 387.5(1200) ;{ }^{1} \mathrm{H} \mathrm{NMR}$ $\left(\mathrm{CDCl}_{3}\right) \delta: 5.00\left(\mathrm{~s}, 2 \mathrm{H}, \mathrm{CH}_{2}\right), 7.3-7.5(\mathrm{~m}, 5 \mathrm{H}), 7.78(\mathrm{~d}, 1 \mathrm{H}, J=2.3 \mathrm{~Hz}, 4-\mathrm{H}), 8.43(\mathrm{~d}, 1 \mathrm{H}, J=$ $2.3 \mathrm{~Hz}, 6-\mathrm{H}) ;{ }^{13} \mathrm{C}$ NMR $\delta: 42.8,112.2,127.8,128.2,128.7,128.9,132.4,132.50,154.1,157.6$, 161.3, 181.0; MS m/z (\%) = 272( $\left.\mathrm{M}^{+}, 19\right), 274\left(\mathrm{M}^{+}+2,5\right), 244(1), 246(1), 215$ (19), $217(5), 181$ (43), 183 (14), 125 (5), 127 (1), 91 (100); HRMS Calcd for $\mathrm{C}_{14} \mathrm{H}_{9} \mathrm{~N}_{2} \mathrm{O}_{2} \mathrm{Cl} 272.0351$ found 272.0324 and 274.0322 found 274.0320 .

\section{Acknowledgements}

This work was partly supported by AIT Special Grant for Education and Research.

\section{References}

1. (a) Popowycz, F.; Routier, S.; Joseph, B.; Mérour, J.-Y. Tetrahedron 2007, 63, 1031. (b) Merour, J.-Y.; Joseph, B. Curr. Org. Chem. 2001, 5, 471.

2. Kägi, H. Helv. Chim. Acta 1941, 24, 141E.

3. (a) Parrick, J.; Yahya, A.; Jin, Y. Tetrahedron Lett. 1984, 25, 3099. (b) Parrick, J.; Yahya, A.; Ijaz, S. A.; Jin, Y. J. Chem. Soc., Perkin Trans. I 1989, 2009.

4. Valentine,J. J.; Nakanishi, S., Hageman, D. L.; Snider, R. M.; Spencer, R. W.; Vinick, F. J. Bioorg. Med. Chem. Lett. 1992, 2, 333.

5. (a) Tatsugi, J.; Zhiwei, T.; Izawa, Y. ARKIVOC 2001, (1), 67. (b) Tatsugi, J.; Zhiwei, T.; Amano, T.; Izawa, Y. Heterocycles 2000, 53, 1145.

6. (a) Tatsugi, J.; Ikuma, K.; Izawa, Y. Tetrahedron Lett. 1995, 36, 8611. (b) Tatsugi, J.; Ikuma, K.; Izawa, Y. Heterocycles 1996, 43, 7. (c) Tatsugi, J.; Hara, T.; Izawa, Y. Chem. Lett. 1997, 177.

7. Tatsugi, J.; Okumura, S.; Izawa, Y. Bull. Chem. Soc. Jpn. 1986, 59, 3311.

8. Tatsugi, J.; Izawa, Y. J. Chem. Res. (S), 1988, 356.

9. Tatsugi, J.; Izawa, Y. Synth. Commun.1998, 28, 859.

10. We examined a comparative study between bromination of 1a with NBS in DMF and that of 1a with bromine in acetic acid under similar conditions. The use of bromine in acetic acid as 
bromination method gave 2a in low yield. We concluded that the system reported here NBS in DMF method on bromination of $1 \mathbf{a}$ is better than the use of $\mathrm{Br}_{2} / \mathrm{AcOH}$. 